\title{
RESIDUAL FINITENESS OF COLOR LIE SUPERALGEBRAS
}

\author{
YU. A. BAHTURIN AND M. V. ZAICEV
}

\begin{abstract}
A (color) Lie superalgebra $L$ over a field $K$ of characteristic $\neq 2,3$ is called residually finite if any of its nonzero elements remains nonzero in a finite-dimensional homomorphic image of $L$. In what follows we are looking for necessary and sufficient conditions under which all finitely generated Lie superalgebras satisfying a fixed system of identical relations are residually finite. In the case char $K=0$ we show that a variety $V$ satisfies this property if and only if $V$ does not contain all center-by-metabelian algebras and every finitely generated algebra of $V$ has nilpotent commutator subalgebra.
\end{abstract}

\section{INTRODUCTION}

Many authors have considered a natural generalization of the class of Lie algebras called color Lie superalgebras [1, 2, 3]. We recall that, given a field $F$ of characteristic different from 2 , an abelian group $G$, and an alternating bilinear form $\varepsilon: G \times G \rightarrow F^{*}$, i.e.,

$$
\varepsilon(g+h, k)=\varepsilon(g, k) \varepsilon(h, k), \quad \varepsilon(g, h)=\varepsilon(h, g)^{-1},
$$

we call a $G$-graded algebra $L=\sum_{g \in G} L_{g}$ an $(\varepsilon$-)color Lie superalgebra if for any $x \in L_{g}, y \in L_{h}$, and $z \in L$ we have

$$
\begin{gathered}
{[x, y]=-\varepsilon(g, h)[y, x],} \\
{[[x, y], z]=[x,[y, z]]-\varepsilon(g, h)[y,[x, z]] .}
\end{gathered}
$$

If $\varepsilon$ is trivial, i.e., $\varepsilon(g, h)=1$ for all $g, h \in G$, then $L$ becomes an ordinary $G$-graded Lie algebra. If $G=Z_{2}$ and $\varepsilon(1,1)=-1$, then we arrive at ordinary Lie superalgebras.

The aim of the present paper is to discuss the question about residual finiteness of color Lie superalgebras. Here we say that a color Lie superalgebra $L=\sum_{g \in G} L_{g}$ is residually finite if for any $x \neq 0, x \in L$, there exists a homomorphism $\phi: L \rightarrow M=\sum_{g \in G} M_{g}$ onto a finite-dimensional color superalgebra such that $\phi(x) \neq 0$. It is assumed here that $\phi$ is homogeneous, that is, we have $\phi\left(L_{g}\right) \subset M_{g}$ for all $g \in G$. An example of a color Lie superalgebra which is not residually finite is given by the Heisenberg superalgebra $\Gamma=\Gamma(g, h)$, where $g, h \in G$. The basis of this superalgebra is formed by the set $\left\{a_{i}, b_{i}, z \mid i \in \mathbf{Z}\right\}$ and $\Gamma_{\alpha}=0$ for $\alpha \neq g, h, g+h, a_{i} \in \Gamma_{g}, b_{i} \in \Gamma_{h}(i \in \mathbf{Z}), z \in \Gamma_{g+h}$. The commutator is given by the formula

$$
\left[a_{i}, b_{i}\right]=-\varepsilon(g, h)\left[b_{i}, a_{i}\right]=z, \quad i \in \mathbf{Z},
$$

Received by the editors August 1, 1989 and, in revised form, May 1, 1990 and January 11, 1991. 1991 Mathematics Subject Classification. Primary 17B65, 17A70. 
with all other commutators being zero. It is easy to see that $\Gamma$ is not residually finite since the image of $z$ under any homomorphism onto a finite-dimensional superalgebra is trivial.

To obtain an example of a finitely generated color Lie superalgebra it is necessary to adjoin to $\Gamma(g, h)$ a binding derivation $d: \Gamma(g, h) \rightarrow \Gamma(g, h)$ and then to place it into the zero component of the newly born algebra. As a result, we obtain a superalgebra $B=B(g, h)$ all of whose components, except zero, are the same as in $\Gamma(g, h)$ and $B_{0}=\Gamma_{0} \oplus\langle d\rangle$, with commutator given by:

$$
\begin{aligned}
& {\left[d, a_{i}\right]=\varepsilon(g, h) a_{i+1}-\varepsilon(h, g) a_{i-1},} \\
& {\left[d, b_{j}\right]=\varepsilon(h, g) b_{j+1}-\varepsilon(g, h) b_{j-1} .}
\end{aligned}
$$

We ask the reader to verify that each of the algebras $B(g, h)$ is indeed an $(\varepsilon-)$ color Lie superalgebra, that it is finitely generated, and that it is not residually finite (since it contains $\Gamma(g, h))$.

Any $B(g, h)$ is center-by-metabelian in the sense that it satisfies an identical relation of the form

$$
[x,[y, z],[u, v]]=0,
$$

where $x, y, z, u$, and $v$ are arbitrary variables and we are using right-normed notation. Our first result is

Theorem 1. Any finitely generated metabelian color Lie superalgebra with finite grading group is residually finite.

The following example shows that we cannot extend Theorem 1 to infinite grading groups. For this we consider a metabelian Lie algebra $L=P \oplus M$, where $P$ is abelian with basis $\{x, y\}$ and $M$ is abelian with basis $\left\{z_{i} \mid i \in \mathbf{Z}\right\}$. We set $\left[x, z_{i}\right]=z_{i-1}$ and $\left[y, z_{i}\right]=z_{i+1}, i \in \mathbf{Z}$. Now $L$ is Z-graded if $L_{i}=\left\langle z_{i}\right\rangle$ for all $i \neq+1,-1, L_{-1}=\left\langle x, z_{-1}\right\rangle$, and $L_{1}=\left\langle y, z_{1}\right\rangle$. If we set $\varepsilon(m, n)=1$ for all $m, n \in \mathbf{Z}$, then $L$ becomes a color Lie superalgebra, and $M$ becomes the minimal nonzero graded ideal of $L$, i.e., its monolith. Thus, $L$ is a 3-generator monolithic metabelian algebra of infinite dimension, hence not residually finite.

In fact Theorem 1 is a particular case of a theorem which is formulated by using the following notation. We denote by $G_{+}$the set of elements $g$ in the grading group $G$ such that $\varepsilon(g, g)=1$. Similarly, $G_{-}=\{g \in G \mid \varepsilon(g, g)=$ $-1\}$. Then $G=G_{+} \cup G_{-}$and $G_{+}$is a subgroup of $G$. We also write

$$
L_{+}=\sum_{g \in G_{+}} L_{g}, \quad L_{-}=\sum_{g \in G_{-}} L_{g} .
$$

If we use this notation then the following holds.

Theorem 2. Let $F$ be a field, char $F \neq 2, G$ be a finite group, and $L$ be a finitely generated color Lie superalgebra with an abelian ideal $A$ such that $L / A$ is finite-dimensional and, in addition, if char $F=0$, we assume that $L_{+}^{2} \subset A$. Then $L$ is residually finite.

The largest portion of this paper is devoted to varieties of color Lie superalgebras. As usual, by a variety we mean a class of superalgebras (with $G$ and $\varepsilon$ 
fixed) satisfying a fixed system of identities. Now, given a $G$-graded alphabet $X=\bigcup_{g \in G} X_{g}$, an equation of the form

$$
f\left(x_{1}, \ldots, x_{n}\right)=0 \quad\left(x_{i} \in X_{g_{i}}, g_{i} \in G, i=1, \ldots, n\right),
$$

where $f\left(x_{1}, \ldots, x_{n}\right)$ is a (nonassociative, commutator) polynomial with coefficients in $F$, is called an identity in a color Lie superalgebra $L$ if we have $f\left(a_{1}, \ldots, a_{n}\right)=0$ for any choice of $a_{1}, \ldots, a_{n} \in A$ such that $a_{i} \in A_{g_{i}}$, $i=1, \ldots, n$.

We discuss locally residually finite varieties, i.e., in which all finitely generated superalgebras are residually finite. It is obvious that no such variety $\mathscr{V}$ contains an algebra of the form $B(g, h)$. We will show (Theorem 3 ) that any locally residually finite variety $\mathscr{V}$ over an infinite field satisfies a system of identities of the form

$$
\left[x, y^{(n)}, z\right]=\sum_{j=1}^{n} \alpha_{j}\left[y^{(j)}, x, y^{(n-j)}, z\right], \quad x \in X_{g}, y \in X_{0}, \quad z \in X_{h} .
$$

Here $\left[y^{(k)}, x\right]$ stands for the right-normed commutator $[y, \ldots, y, x]$ with $k$ entries of $y$. A surprising fact is that if such a system, for all $g, h$, is satisfied in a variety $\mathscr{V}$ of a locally soluble Lie superalgebras over an infinite field $F$ of positive characteristic, then $\mathscr{V}$ is residually finite (Theorem 4 ). In the case when $F$ is of characteristic zero, for a variety $\mathscr{V}$ of Lie superalgebras to be residually finite it is necessary and sufficient to require in addition to the identities (5) that the commutator $L_{0}^{2}$ of the even component $L_{0}$ of any finitely generated Lie superalgebra $L=L_{0} \oplus L_{1}$ in $\mathscr{V}$ acts on the whole of $L$ as a nilpotent space of transformations (Theorem 5).

We remark that all of the results in this paper are a natural generalization of certain results about ordinary Lie algebras in $[4,5,6]$.

\section{SUFFICIENT CONDITIONS FOR RESIDUAL FINITENESS}

In this section we prove Theorem 2 stated in the Introduction. We recall that the enveloping algebra $U(L)$ is a $G$-graded associative algebra $A$ with imbedding $l: L \rightarrow A$ such that

$$
l([x, y])=l(x) l(y)-\varepsilon(g, h) l(y) l(x),
$$

where $x \in L_{g}$ and $y \in L_{h}$, and such that if $\tau: L \rightarrow B$ is a similar homomorphism into a $G$-graded associative algebra $B$, then there exists a unique homogeneous homomorphism $f: A \rightarrow B$ such that $f(l(x))=\tau(x)$ for all $x \in L$.

It is known (in the case of ordinary Lie superalgebras of characteristic zero see, for example, [7, p. 26]) that if $F$ is a field, then $l$ is a monomorphism which enables us to identify $L$ with a subalgebra in $A$ (under the operation

$$
[x, y]=x y-\varepsilon(g, h) y x,
$$

$\left.x \in A_{g}, y \in A_{h}\right)$. Moreover, if $E=\bigcup_{g \in G} E_{g}$ is a totally ordered basis of $L$ such that $E_{g}$ is a basic of $L_{g}$, then $U(L)$ is formed by 1 together with all "ordered" monomials of the form

$$
e_{1} e_{2} \cdots e_{n} \quad\left(e_{i} \in E, e_{1} \leq e_{2} \leq \cdots \leq e_{n}\right)
$$


where we cannot have $e_{i}=e_{i+1}$ for $e_{i} \in E_{g}$ with $g \in G_{-}$.

In particular, if $L=L_{+} \oplus L_{-}$is a finite-dimensional color Lie superalgebra, then $U(L)$ is a free left and right module of finite type $2^{s}$ over its subalgebra $U\left(L_{+}\right)$, where $s=\operatorname{dim} L_{-}$. As a vector space, $U(L)$ takes the form

$$
U(L)=S\left(L_{+}\right) \otimes \Lambda\left(L_{-}\right),
$$

where $S(V)$ is the symmetric algebra of the vector space $V$ and $\Lambda(V)$ is the Grassmann algebra for $V$. It is clear that, in general, the equality in (9) is not a homomorphism of algebras. However, following the proofs of well-known results, we can prove

Proposition 1. (1) $U\left(L_{+}\right)$has no zero-divisors.

(2) If $L$ is finite dimensional, then $U(L)$ is noetherian.

(3) If $L$ is finite dimensional and abelian and $G$ is finite, then any irreducible $L$-module is finite dimensional.

(4) If $L$ is finite dimensional, $G$ is finite, and char $F=p>0$, then any irreducible L-module is finite dimensional.

Proof. We have a filtration in $U(L)$ of the form

$$
\{0\}=U_{-1} \subset U_{0} \subset U_{1} \subset \cdots \subset U_{n} \subset \cdots,
$$

where $U_{n}$ is the linear span of all products of elements in $L$ with at most $n$ factors. The associated graded algebra is the enveloping algebra for the abelian algebra $\bar{L}$ with basis $E=\bigcup_{g \in G} E_{g}$. Therefore, $U(\bar{L})$ is generated by $E$ with respect to defining relations of the form

$$
e e^{\prime}=\varepsilon(g, h) e^{\prime} e, \quad \text { where } e \in E_{g}, e^{\prime} \in E_{h} .
$$

It is obvious then that, in $U(L)$, the degree of elements is defined with the usual properties. If we restrict to the subalgebra generated by $E_{+}=\bigcup_{g \in G_{+}} E_{g}$, then the degree of the product is equal to the sum of the degrees of the factors (more exactly, that the lexicographically leading term of the product is the product of the lexicographically leading terms of the factors). Now (1) follows since $E_{+}$ generates $U\left(\bar{L}_{+}\right)=\operatorname{gr} U\left(L_{+}\right)$.

It is sufficient to verify the noetherian property for $U\left(L_{+}\right)$only, since $U(L)$ is of finite type over $U\left(L_{+}\right)$. Again the key point is passing to $U(\bar{L})$. It is easy to see that, under our hypotheses, we can apply for our proof the usual procedure as found in the proof of Hilbert's Basis Theorem with induction over the number of variables $\operatorname{dim} \bar{L}_{+}$.

Let $V$ be an irreducible (graded) $L$-module, $L$ being a Lie superalgebra with finite grading group $G$ and with form $\varepsilon: G \times G \rightarrow F^{*}$. Then $V$ is a left module over $U(L)=U$. Every nonzero component $V_{h}$ is obviously an irreducible $U_{0}$-module. Since $G$ is finite it is sufficient to establish that any irreducible (nongraded) $U_{0}$-module is finite dimensional. If $n=|G|$ then, clearly, any $\varepsilon(g, h)$ is an $n$th root of unity. In this case, given a basis $E=\left\{e_{1}, \ldots, e_{m}\right\}$ of $L$, the elements $e_{1}^{2 n}, \ldots, e_{m}^{2 n}$ generate a central polynomial subalgebra $Z$ such that $U_{0}$ is a free (left, right) $Z$-module of finite type. For, if $e_{i} \in L_{g}$ and $e_{j} \in L_{h}$, we have

$$
\begin{aligned}
e_{i}^{2 n} e_{j} & =\varepsilon(g, h) e_{i}^{2 n-1} e_{j} e_{i}=\varepsilon^{2}(g, h) e_{i}^{2 n-2} e_{j} e_{i}^{2} \\
& =\cdots=\varepsilon^{2 n}(g, h) e_{j} e_{i}^{2 n}=\varepsilon(2 n g, h) e_{j} e_{i}^{2 n}=e_{j} e_{i}^{2 n} .
\end{aligned}
$$


By Curtis' theorem [8] all irreducible representations of $U_{0}$ are finite dimensional, proving (3).

Finally, let $L$ be a finite-dimensional Lie superalgebra over a field $F$ of characteristic $p, p>0, x \in L_{g}, g \in G_{+}$, and $f$ a $p$-polynomial annihilating ad $x$. Then $f(\operatorname{ad} x)=\operatorname{ad} f(x)$. For this, it is useful to notice that, given $u \in U(L), u=u_{g_{1}}+\cdots+u_{g_{k}}, v \in U(L)_{h}$, we have

$$
(\operatorname{ad} u)(v)=\left(u_{g_{1}} v-\varepsilon\left(g_{1}, h\right) v u_{g_{1}}\right)+\cdots+\left(u_{g_{k}} v-\varepsilon\left(g_{k}, h\right) v u_{g_{k}}\right) .
$$

Now if $x \in L_{g}, g \in G_{+}$, and $y \in L_{h}$ then

$$
(\operatorname{ad} x)^{p}(y)=\left(L_{x}-\varepsilon(g, h) R_{x}\right)^{p}(y)=\left(L_{x^{p}}-\varepsilon(p g, h) R_{x^{p}}\right)(y)=\left(\operatorname{ad} x^{p}\right)(y) .
$$

Here, under the consecutive actions of ad $x$ the value of the form $\varepsilon$ does not change since on the $(k+1)$ th step ad $x$ transforms $(\operatorname{ad} x)^{k}(y) \in L_{k g+h}$ and

$$
\varepsilon(g, k g+h)=\varepsilon(g, g)^{k} \varepsilon(g, h)=\varepsilon(g, h) .
$$

It is easy to observe that if $u=f(x)=u_{g_{1}}+\cdots+u_{g_{k}}$ is an element with the property ad $f(x)=0$ and $g_{i} \neq g_{j}$ for $i \neq j$, then ad $u_{g_{1}}, \ldots$, ad $u_{g_{k}}$ have the same property. Now if we take $u_{g_{1}}^{n}, \ldots, u_{g_{k}}^{n}$ with $n=|G|$, then these elements belong to the usual center of $U(L)$. The subalgebra $Z$ generated by all $u_{g_{1}}^{n}, \ldots, u_{g_{k}}^{n}$ with $x$ running through the basis $\left\{e_{1}, \ldots, e_{r}\right\}$ of $L_{+}$is in the center of $U(L)$, which is a module of finite type over $Z$. Now the same argument as before proves the finiteness of the dimension of irreducible $L$-modules. The proof of the proposition is complete.

Now we are able to pass to the proof of Theorem 2 stated in $\S 1$. This is quite similar to the proof in [4].

Proof of Theorem 2. Let $g_{1}, \ldots, g_{t}$ be a generating system of $L$ such that $g_{1}+A, \ldots, g_{t}+A$ contains a basis of $H=L / A$. By a linear change of variables we can assume that some $\left\{g_{1}+A, \ldots, g_{s}+A\right\}$ is a basis in $L / A$ and $g_{s+1}, \ldots, g_{t} \in A$. Let $\left\{c_{i j}^{k}\right\}$ be the set of structure constants with respect to the given basis. For any $i, j \quad(1 \leq i, j \leq s)$ we set

$$
r_{i j}=\left[g_{i}, g_{j}\right]-\sum_{k} c_{i j}^{k} g_{k} .
$$

It is obvious that $r_{i j} \in A$. If $J=\operatorname{id}_{L}\left\{r_{i j}, g_{s+1}, \ldots, g_{t}\right\}$, then $A \supset J$. On the other hand, $\operatorname{dim} L / J \leq s=\operatorname{dim} L / A$. Then $A=J$, i.e., $A$ is the ideal generated by $\left\{r_{i j}, g_{s+1}, \ldots, g_{t} \mid 1 \leq i, j \leq k\right\}$.

Now we set $H=L / A$. Then $A$ is a finitely generated $H$-module with respect to the adjoint action. According to the above proposition, $U(H)$ is a noetherian algebra, hence, $A$ is a noetherian module. For the proof of the residual finiteness of $L$ it is sufficient to verify the finiteness of the dimension of its monolithic homomorphic images, i.e., those which have the least nonzero ideal, the monolith. Without any loss of generality we can assume that already $L$ itself is monolithic, and $M$ is its monolith. According to claims (3) and (4) in the above proposition, its monolith $M$ is a finite-dimensional space. Hence the proof of the theorem will be complete if we manage to prove the following.

Lemma 1. Let $L$ be a finitely generated color Lie superalgebra with an abelian ideal $A$ satisfying the hypotheses of Theorem 2 , and let $M$ be a 
finite-dimensional (homogeneous) ideal in $L$ having nonzero intersection with any other ideal $K$ in $L, K \neq 0$. Then $L$ is finite dimensional.

Proof. Let $H=L / A$. If we consider the proof of Proposition 1 then we see that $U(H)$ has a central noetherian $G$-homogeneous subalgebra $Z$ such that $U(H)$ is a $Z$-module of finite type. Let $z$ be an arbitrary $G$-homogeneous element in $Z \cap$ Ann $M$. Then the chain of subspaces of the form

$$
z A \supset z^{2} A \supset \cdots \supset z^{t} A \supset \cdots
$$

is, in fact, a chain of $U(H)$-submodules. Let us assume that none of the subspaces in the chain (11) is zero; in this case for any $t$ we also have $z^{t} A \cap M \neq 0$. We consider also the chain

$$
M \cap A \subset \operatorname{Ann}_{A} z \subset \operatorname{Ann}_{A} z^{2} \subset \cdots \subset \operatorname{Ann}_{A} z^{u} \subset \cdots .
$$

Since $A$ is a noetherian $U(H)$-module, we have, for a suitable $u$, the following:

$$
\mathrm{Ann}_{A} z^{u}=\mathrm{Ann}_{A} z^{u+1}=\cdots .
$$

We consider $m \in M \cap z^{u} A, m \neq 0$. Then, for some $a \in A$, we have $m=$ $z^{u} a$. Furthermore, $0=z m=z\left(z^{u} a\right)=z^{u+1} a$, i.e., $a \in \operatorname{Ann}_{A} z^{u+1}$. It follows from (13) that then $z^{u} a=0$, hence also $m=0$, a contradiction. Hence in the chain (11) not all the spaces are nonzero, that is, the action of $z$ on $A$ is nilpotent. Now if $b_{1}, \ldots, b_{k}$ are the $Z$-generators of $U(H)$, and $z_{1}, \ldots, z_{l}$ the generators of $Z$, then there exist polynomials $f_{i}$ so that $u_{1}=$ $f_{1}\left(z_{1}\right), \ldots, u_{l}=f_{l}\left(z_{l}\right)$ are elements in $Z \cap$ Ann $M$. Hence any element in $U(H)$ can be represented as a linear combination of monomials of the form

$$
b_{i} z_{1}^{k_{l}} \cdots z_{l}^{k_{l}} u_{1}^{t_{1}} \cdots u_{l}^{t_{l}}, \quad 0 \leq k_{i}<d_{i}, d_{i}=\operatorname{deg} f_{i}, i=1, \ldots, l, t_{i} \geq 0 .
$$

If $n_{i}$ is the nilpotent index for the action of $u_{i}$ on $A$ then only a finite number of elements of the form (14), with $t_{i}<n_{i}$ for all $i=1, \ldots, l$, can act on $A$ in a nonzero way. Since $A$ is finitely generated, we deduce that it is finite dimensional. It follows by $\operatorname{dim} L / A<\infty$ that also $\operatorname{dim} L<\infty$. Now the proof of Lemma 1 and, with it, the proof of Theorem 2 is complete.

\section{IDENTICAL RELATIONS IN LOCALLY RESIDUALLY FINITE VARIETIES}

Theorem 3. Let $\mathscr{V}$ be a locally residually finite variety of color Lie superalgebras over an infinite field (with respect to a form $\varepsilon: G \times G \rightarrow F^{*}$ ). Then for any $g, h \in G$ an identity of the following form holds in $\mathscr{V}:$

$$
\left[x, y^{(n)}, z\right]=\sum_{j=1}^{n} \alpha_{j}\left[y^{(j)}, x, y^{(n-j)}, z\right],
$$

where $\alpha_{j} \in F, j=1, \ldots, n, x \in X_{g}, y \in X_{0}$, and $z \in X_{h}$.

Proof. Since $F$ is infinite, it is sufficient to restrict to multihomogeneous identities only. It is known [9] that a variety of ordinary Lie algebras which does not contain $B(0,0)$ consists of algebras satisfying (15). It follows that $\mathscr{V}$ satisfies (15) with $x, y, z \in X_{0}$. To prove (15) in the case $g=0$ we consider $B=B(0, h)$, where $h \neq 0$. We have

$$
B_{0}=\left\langle d, a_{i}(i=\cdots-1,0,1, \ldots)\right\rangle, \quad B_{h}=\left\langle z, b_{i}(i=\cdots-1,0,1, \ldots)\right\rangle .
$$


Since $B$ is not locally residually finite, $\mathscr{V} \not \supset B$. Any multihomogeneous identity with variable $x \in X \backslash\left(X_{0} \cup X_{h}\right)$ is satisfied in $B$. Hence $\mathscr{V}$ must satisfy a multihomogeneous identity in the variables of $X_{0} \cup X_{h}$ which is not satisfied by $B$. Since $B_{h}$ is an abelian ideal, $B$ satisfies any multihomogeneous identity depending on at least two variables in $X_{h}$. Assume first that there is no identity with one variable in $X_{h}$ which is satisfied in $\mathscr{V}$ but does not hold in $B$. Now since $B_{0}$ is a metabelian algebra, any multihomogeneous identity in the variables of $X_{0}$ satisfied by $\mathscr{V}$ but not $B$ can be brought to the form

$$
\sum_{k=1}^{n} \beta_{k}\left[x_{1}, \ldots, x_{k-1}, x_{k+1}, \ldots, x_{n}, x_{k}, x_{0}\right] \equiv 0
$$

modulo the metabelian identity, where like terms are reduced. (Note that this identity as well as some others to follow are not assumed multilinear.) If, say, $\beta_{1} \neq 0$, then setting $x_{1}=y+z, x_{i}=y$ if $x_{i} \neq x_{1}$, and taking the sum of monomials of degree 1 in $z$ we get $\left[y^{(n)}, z\right] \equiv 0$. It is obvious that the same substitution in each of the consequences of $[[x, y],[z, u]] \equiv 0$ gives zero. Now, clearly, $\left[y^{(n)}, z\right] \equiv 0$ implies (15) with all $\alpha_{j}=0:\left[x, y^{(n)}, z\right] \equiv 0$ with $x \in X_{h}$. If we want to have (15) with $z \in X_{h}$ then we know already that $\left[z, y^{(n)}, x\right] \equiv 0$, hence $\left[\left[y^{(n)}, x\right], z\right] \equiv 0$. Since we have

$\left[(\operatorname{ad} y)^{n}, \operatorname{ad} x\right]=\left(L_{\mathrm{ad} y}-R_{\mathrm{ad} y}\right)^{n}(\operatorname{ad} x)=\sum_{k=0}^{n}(-1)^{k}\left(\begin{array}{l}n \\ k\end{array}\right)(\operatorname{ad} y)^{n-k}(\operatorname{ad} x)(\operatorname{ad} y)^{k}$,

it follows that

$$
\left[\left[y^{(n)}, x\right], z\right]=\sum_{k=0}^{n}(-1)^{k}\left(\begin{array}{l}
n \\
k
\end{array}\right)\left[y^{(n-k)}, x, y^{(k)}, z\right],
$$

which gives (15) with $x, y \in X_{0}$ and $z \in X_{h}$.

Now suppose $\mathscr{V}$ satisfies an identity with one variable in $X_{h}$ which is not satisfied in $B(0, h)$. Since $B(0, h)$ satisfies

$$
\left[x_{1},\left[x_{2}, x_{3}\right], z\right]=0, \quad x_{i} \in X_{0}, \quad z \in X_{h},
$$

our additional identity is not a consequence of (16). We reduce that identity modulo (16). Then it takes the form

$$
\sum_{k=1}^{n+1} \gamma_{k}\left[x_{k}, x_{1}, \ldots, x_{k-1}, x_{k+1}, \ldots, x_{n}, z\right] \equiv 0 \text {. }
$$

Let $\gamma_{1} \neq 0$. Then, setting $x_{1}=x+y, x_{i}=y$ if $x_{i} \neq x_{1}$, and taking the sum of monomials of degree 1 in $x$ yields

$$
\gamma_{1}\left[x, y^{(n)}, z\right]+\left(\sum_{k=2}^{n+1} \gamma_{k}\right)\left[y, x, y^{(n-1)}, z\right] \equiv 0 \text {. }
$$

On the other hand, if we apply the same substitution in the consequences of (16), we get terms on the right-hand side of (15), proving that, indeed, $\mathscr{V}$ satisfies the identities of the form as claimed.

To get (15) of arbitrary form we consider $B=B(g, h)$ with $g, h \neq 0$. Since $B$ is not residually finite we have $B \notin \mathscr{V}$. As before, $\mathscr{V}$ must satisfy 
an identity in the variables of $X_{0} \cup X_{g} \cup X_{h} \cup X_{g+h}$. If $g+h \neq 0$ then $B_{g+h}=\langle z\rangle$ and the only identity including a variable $u \in X_{g+h}$ which does not hold in $B$ is $u \equiv 0$. However, it follows from this identity that $[x, z]=0$ with $x \in X_{g}$ and $z \in X_{h}$, and this implies $[x, y, z]=0$ with $x \in X_{g}, y \in X_{0}$, and $z \in X_{h}$, i.e., the identity as required. Hence, we may assume that the identities of $\mathscr{V}$ which do not hold in $B$ include only the variables in $X_{0} \cup X_{g} \cup X_{h}$. It is very clear that an "additional" identity cannot contain more than two variables in $X_{g} \cup X_{h}$ since $B_{g}+B_{h}+\langle z\rangle$ is a 2-step nilpotent ideal in $B$. If this identity depends only on variables in $X_{0}$ then it must be $y \equiv 0$, with $y \in X_{0}$, which, obviously implies (15). If the additional identity contains only one variable which is not in $X_{0}$, say, $z \in X_{h}$, then, since we have $\left[y_{1}, y_{2}\right]=0$ in $B$ for $y_{1}, y_{2} \in X_{0}$, this identity is equivalent to $\left[y_{1}, \ldots, y_{n}, z\right]=0$. If we identify all $y_{1}, \ldots, y_{n}$ with some $y$ we have $\left[y^{(n)}, z\right]=0$, hence $\left[x, y^{(n)}, z\right] \equiv 0$, hence $(15)$. Now let the additional identity contain two variables $x \in X_{g}$ and $z \in X_{h}$ with all the rest in $X_{0}$. Since $B=B(g, h)$ satisfies

$$
\begin{aligned}
& {[y, x, z]=0, \quad y \in X_{0}, x \in X_{g}, \quad z \in X_{h},} \\
& {\left[y_{1}, y_{2}\right]=0, \quad y_{1}, y_{2} \in X_{0},}
\end{aligned}
$$

our additional identity reduces, modulo (17), to the form

$$
\left[x, y_{1}, \ldots, y_{n}, z\right] \equiv 0 \text {. }
$$

If we identify $y_{1}, \ldots, y_{n}$ then we get $\left[x, y^{(n)}, z\right] \equiv 0$. As for the consequences of the first identity in (17), either these are zero or else they have the form of a linear combination of monomials of the form $\left[y,\left[y^{(m)}, x\right],\left[y^{(k)}, z\right]\right], m+k=$ $n-1$; if $g+h=0$, the consequences of the second identity in (17) have the same form, and if $g+h \neq 0$ then all the consequences of the second identity are zero. It is clear the rewriting these monomials in the right-normed form gives only the monomials in the right-hand side of (15), proving the theorem.

\section{TWo LEMMAS ON REPRESENTATIONS OF SOLUbLE LIE ALGEBRAS}

This section contains some auxiliary results. The motivation for the lemmas that follow lies in the fact that, given an arbitrary color Lie superalgebra $L=$ $\sum_{g \in G} L_{g}$, any of its components $L_{g}$ becomes an $L_{0}$-module, where $L_{0}$ is an ordinary Lie algebra.

Lemma 2. Let $L$ be a finitely generated soluble Lie algebra over an infinite field $F$, and let $V$ be an $L$-module. If a representation of $L$ by linear operators of $a$ vector space $V$ satisfy identities of the form

$$
\begin{gathered}
{\left[y^{(n)}, x\right]=\sum_{j=1}^{n} \lambda_{j} y^{j}\left[y^{(n-j)}, x\right],} \\
{\left[z, y^{(n)}, x\right]=\sum_{j=1}^{n} \alpha_{j}\left[y^{(j)}, z, y^{(n-j)}, x\right],}
\end{gathered}
$$

then, for some $m, L^{m}$ acts on $V$ as a nilpotent space of transformations, where $L^{m}=[L, L, \ldots, L]$ with $m$ factors.

Proof. We apply to (18) the method of divided variables due to Mishchenko [10]. If $\lambda_{j}=0$ for all $j=1, \ldots, n$ in $(18)$, then $g(x, y)=\left[y^{(n)}, x\right]$ is 
equal to zero on $V$ identically. Since $F$ is an infinite field, the linear span of values of $g$ is an ideal. This ideal contains $L^{m}$ for some $m$ because any finitely generated soluble Lie algebra with Engel condition is nilpotent (see, for example, [11, Theorem 4.7.2]).

Let $s$ be the greatest of the indices in (18) such that $\lambda_{s} \neq 0$ and $z, y \in L$. We consider the operators $A, B, C, D: L \rightarrow$ End $V$ such that for all $x \in L$ we have

$$
A(x)=y x, \quad B(x)=[y, x], \quad C(x)=z x, \quad D(x)=[z, x] .
$$

Then (18) can be written in the form $f B^{n-s}=0$ on $L$, where

$$
f=f(A, B)=B^{s}-\sum_{j=1}^{s} \lambda_{j} A^{j} B^{s-j} .
$$

Now let $f_{i}$ be the sum of monomials of total degree $i$ in $C, D$ in the polynomial $f(A+C, B+D)$. Then $f_{0}=f(A, B)$ and $f_{s}=f(C, D)$. We will show that for each $i=0, \ldots, s$ there exists a number $k_{i}$ such that

$$
f_{i} B^{k_{i}}=0
$$

on $L$.

If $i=0$ then such a relation follows from (18) if we set $k_{0}=n-1$. Now we assume that the numbers $k_{0}, k_{1}, \ldots, k_{i-1}$ have been found. For any nonnegative $t$ we have by (19) an equation of the form

$$
f(A+C, B+D)\left[(y+z)^{(n-s)}, y^{(t)}, x\right]=0 .
$$

Since $F$ is infinite, the sum of monomials of degree $i$ in $z$ in (21) is also trivial. It takes the form

$$
f_{i}\left[y^{(n-s+t)}, x\right]+\sum_{j} f_{j} g_{j}(\operatorname{ad} y, \text { ad } z)(x) .
$$

Here the summation over $j$ goes from 0 to $i-1$ if $i \leq n-s$ and from $i-n+s$ to $i-1$ if $i>n-s$, and $g_{j}$ is a homogeneous polynomial of degree $i-j$ in ad $z$ and of degree $d=n-s+t-i+j$ in ad $y$. It follows from (19) that $g_{j}(\operatorname{ad} y, \operatorname{ad} z)$ has the form $(\operatorname{ad} y)^{k_{j}} h_{j}(\operatorname{ad} y$, ad $z)$ if $d \geq k_{j}+n(i-j)$, i.e., $t \geq r_{i j}=k_{j}+n(i-j-1)+i-j+s$. Now it is sufficient to define $k_{i}$ as the greatest of the numbers $r_{i j}$. Since the homogeneous component of degree $i$ in $z$ is equal to zero in (21), we have (20). If $i=s$, then (20) takes the form

$$
\left[z^{(s)}, T\right]=\sum_{j=1}^{s} \lambda_{j} z^{j}\left[z^{(s-j)}, T\right],
$$

where $T=\left[y^{(k)}, x\right]$ for suitable $k$ and where the last summand in the righthand side takes the form $\lambda_{s} z^{s} T$ with $\lambda_{s} \neq 0$. Since $F$ is an infinite field the linear span of values of $T$ is an ideal, while it follows from the local nilpotence of soluble Engel Lie algebras that this ideal contains $L^{m}$ for some $m$. Having substituted $z=T=b \in L^{m}$ in (23), we get the equation of the form $b^{s+1}=0$.

Now we want to prove that $L^{m}$ acts on $V$ as a nilpotent space of transfomations. It was proved in [6] that any finitely generated soluble Lie algebra with an identity of the form (19) is in the product of two nilpotent varieties (Lemma 
6). Hence, without loss of generality, we may assume that $L^{m}$ is a nilpotent Lie algebra.

Since $L$ is finitely generated, $L / L^{m}$ is finite dimensional. Now let $\left\{e_{1}, \ldots\right.$, $\left.e_{q}\right\}$ be a basis of $L$ modulo $L^{m}$ such that $\left[e_{i}, e_{j}\right]$ can be written as a linear combination (modulo $L^{m}$ ) of elements $e_{k}$ with $k<i, j$. Let $a_{1}, \ldots, a_{r}$ be generators of the ideal $L^{m}$ including the elements of the form $\left[e_{i}, e_{j}\right]-$ $\sum_{k=1}^{q} c_{i j}^{k} e_{k}$, where the $c_{i j}^{k}$ are the structure constants of $L / L^{m}$. Using the nilpotence of $L^{m}$ it is easy to verify that, as a Lie algebra, $L^{m}$ can be generated by all commutators of the form

$$
\left[e_{1}^{\left(t_{1}\right)}, \ldots, e_{q}^{\left(t_{q}\right)}, a_{i}\right], \quad t_{1}, \ldots, t_{q} \geq 0, i=1, \ldots, r .
$$

We denote by $H$ the subalgebra in $L^{m}$ generated by elements of the form (24) with $t_{1}, \ldots, t_{q} \leq n-1$. Then $H$ is finite dimensional and acts on $V$ by nilpotent operators. Let $w_{1}, \ldots, w_{q}$ be a basis of $H$. If $v \in V$ then the $H$-submodule $W$ generated by $v$ is a linear span of elements $w_{1}^{r_{1}} \cdots w_{q}^{r_{q}} v$. For any $h \in L^{m}$ and $w \in W$, the element $h^{s+1} w$ is equal to zero. Hence, $\operatorname{dim} W \leq q(s+1)=N$. It is known that $H$ can be represented as a set of upper triangular $(N \times N)$-matrices (see, for example, [11, Chapter 1]). Therefore, $h_{1} \cdots h_{N} v=0$ for any $h_{1}, \ldots, h_{N} \in H$.

For the proof it is sufficient to verify that $b_{1} \cdots b_{N} V=0$ for all $b_{1}, \ldots, b_{N}$ of the form (24). We introduce a partial ordering on the set of such elements by setting

$$
\left[e_{1}^{\left(t_{1}\right)}, \ldots, e_{q}^{\left(t_{q}\right)}, a_{i}\right]<\left[e_{1}^{\left(u_{1}\right)}, \ldots, e_{q}^{\left(u_{q}\right)}, a_{j}\right]
$$

if $\left(t_{1}, \ldots, t_{q}\right)$ is lexicographically less than $\left(u_{1}, \ldots, u_{q}\right)$ (we compare the components from the right to the left). Similarly we can define the ordering of the sets of the form $\left\{b_{1}, \ldots b_{N}\right\}$ by comparing their terms from the right to the left considering the ordering introduced just above.

If all $b_{i}$ in the set $\left\{b_{1}, \ldots, b_{N}\right\}$ are generators of $H$, then $b_{1} \cdots b_{N} v=0$ for any $v \in V$. Otherwise, let $b_{i}$ be the first from right to left generator of $L^{m}$ of the form

$$
b_{i}=\left[e_{1}^{\left(t_{1}\right)}, \ldots, e_{q}^{\left(t_{q}\right)}, a_{j}\right],
$$

where at least one exponent, say $t_{q}$, is not less than $n$.

If $i_{1}>i_{2}$ and $w \in L$ then by the Jacobi identity the commutator $\left[e_{i_{1}}, e_{i_{2}}, w\right]$ is a linear span of elements $\left[e_{i_{3}}, w\right]$, where $i_{3}<i_{2}$, and $\left[e_{i_{2}}, e_{i_{1}}, w\right]$. It follows that if we replace one of the entries of $e_{p}$ in $b_{i}$ by $e_{l}$ with $l<p$ then we get a linear span of some elements $b_{i}^{\prime}$ of the type (24) and $b_{i}^{\prime}<b_{i}$.

If we replace one of the entries of $e_{p}$ in $b_{i}$ by $a_{k}$ then we get a linear span of commutators $\left[d_{1}, d_{2}\right]$, where $d_{1}, d_{2}$ are of the form (24) and $d_{1}, d_{2}<b_{i}$. We set

$$
c=\left[e_{q}^{\left(t_{q}\right)}, e_{1}^{\left(t_{1}\right)}, \ldots, e_{q-1}^{\left(t_{q-1}\right)}, a_{j}\right] .
$$

The commutator $\left[e_{q}, e_{p}\right]$ is equal to $\mu_{1} e_{1}+\cdots+\mu_{p-1} e_{p-1}+a_{k}$ for some $k$, where the $\mu_{1}, \ldots, \mu_{p-1}$ are in $F$. It follows that $b_{i}-c$ is a linear span of some commutators $d$ and $\left[d_{1}, d_{2}\right]$, where $d, d_{1}$, and $d_{2}$ are of the type (24) and less than $b_{i}$.

Now the element $b_{1} \cdots b_{i-1}\left(b_{i}-c\right) b_{i+1} \cdots b_{N} v$ takes the form of a linear combination of products of the form

$$
b_{1} \cdots b_{i-1} d_{j_{1}} d_{j_{2}} b_{i+1} \cdots b_{N} v ; \quad b_{1} \cdots b_{i-1} d_{j} b_{i+1} \cdots b_{N} v
$$


in each of which the "tail" on $N$ rightmost operators, acting on $v$, is strictly less than $\left\{b_{1}, \ldots, b_{N}\right\}$, hence we may apply our induction. So, our element $b_{1} \cdots b_{N} v$ is equal to an element of the form $b_{1} \cdots b_{i-1} c b_{i+1} \cdots b_{N} v$. We set $w=b_{i+1} \cdots b_{N} v$. According to (18), the element $c w$ is equal to a linear combination of the form $e_{q}^{k} c_{k} w$, where

$$
c_{k}=\left[e_{q}^{\left(t_{q}-k\right)}, e_{1}^{\left(t_{1}\right)}, \ldots, e_{q-1}^{\left(t_{q-1}\right)}, a_{j}\right], \quad k>0 .
$$

The vector $b_{1} \cdots b_{i-1} e_{q}^{k} c_{k} w$ is in the $L$-module generated by some elements of the form $d_{1} \ldots d_{i-1} c_{k} w$ with $d_{1}, \ldots, d_{i-1} \in L^{m}$. As all elements (24) generate $L^{m}$ it follows that $b_{1} \cdots b_{i-1} e_{q}^{k} c_{k} w$ is in the $L$-module generated by elements $g_{1} \ldots g_{m} c_{k} w$, where $g_{1}, \ldots, g_{m}$ are of the form (24) and $m \geq i-1$.

Element $c_{k}$ is not of the type (24). If we move $e_{q}$ in $c_{k}$ from left to right using the Jacobi identity and the relation $\left[e_{q}, e_{p}\right]=\mu_{1} e_{1}+\cdots+\mu_{p-1} e_{p-1}+a_{k}$, then we express $c_{k}$ as a linear span of some $f_{k}$ of the form (24) with $f_{k}<b_{i}$. Hence, $b_{1} \cdots b_{i-1} e_{q}^{k} c_{k} w$ is in the $L$-module generated by

$$
g_{1} \cdots g_{m} f_{k} w=g_{1} \cdots g_{m} f_{k} b_{i+1} \cdots b_{N} v .
$$

Since $f_{k}<b_{i}$ and $m \geq i-1$, we may apply induction over the partial ordering on the sets of the form $\left\{b_{1}, \ldots, b_{N}\right\}$. By the inductive hypothesis,

$$
g_{1} \cdots g_{m} f_{k} b_{i+1} \cdots b_{N} v=0
$$

and $b_{1} \cdots b_{N} v=0$.

If $t_{k}>n-1$ for $b_{i}$ with $k \leq q-1$ then the proof is quite similar to the case $k=q$. The proof of Lemma 2 is complete.

Lemma 3. Let $L$ be a finitely generated soluble Lie algebra and $V$ a finitely generated L-module with identities (18) and (19). Then $V$ is a noetherian L-module.

Proof. By Lemma 2 for some $m$ the ideal $L^{m}$ acts on $V$ by nilpotent transformations. Now $V$ possesses a finite series of submodules of the form

$$
V=V_{0} \supset V_{1}=L^{m} V_{0} \supset \cdots \supset V_{k+1}=L^{m} V_{k} \supset \cdots \supset L^{m} V_{N}=\{0\} .
$$

To prove that $V$ is a noetherian $L$-module it is sufficient to verify the noetherian property of each factor $V_{k} / V_{k+1}, k=1, \ldots, N$, where, in fact, each $V_{k} / V_{k+1}$ is an $L / L^{m}$-module. Since $L / L^{m}$ is finite dimensional, $U\left(L / L^{m}\right)$ is noetherian and it is sufficient to verify that each module $V_{k} / V_{k+1}$ is finitely generated.

Let $\left\{e_{1}, \ldots, e_{q}\right\}$ be a basis of $L$ modulo $L^{m}$ and $\left\{a_{1}, \ldots, a_{r}\right\}$ a generating set for $L^{m}$ as an ideal of $L$. The number of commutators of the form

$$
\left[e_{1}^{\left(t_{1}\right)}, \ldots, e_{q}^{\left(t_{q}\right)}, a_{i}\right], \quad 0 \leq t_{1}, \ldots, t_{q} \leq n-1,
$$

is finite. Using induction over $k$ we want to show that $V_{k}=V_{k+1}+P_{k}$, where $P_{k}$ is the $L$-submodule generated by all $b_{1} \cdots b_{k} v$ with each $b_{i}$ of the form (25) and $v$ is one of the generators of $V$ as an $L$-module, the number of such generators being finite. If $k=0$, then it is obvious.

Now let $k \geq 1$ and suppose that $V_{k-1}=V_{k}+P_{k-1}$ is already true. Then $V_{k}=V_{k+1}+H$, where $H$ is the linear span of the set of the elements of the form

$$
d e_{1}^{t_{1}} \cdots e_{q}^{t_{q}} b_{2} \cdots b_{k} v
$$


where $b_{2}, \ldots, b_{k}$ is of the form (25) and $d \in L^{m}$. It follows from this that $V_{k}$, as an $L$-module, is generated by $V_{k+1}$ together with the set of elements of the form $d b_{2} \cdots b_{k} v$, where $d=\left[e_{1}^{\left(t_{1}\right)}, \ldots, e_{q}^{\left(t_{q}\right)}, a_{i}\right]$. If $t_{1}, \ldots, t_{q} \leq n-1$ then $d$ is an element of the form (25) as required. Otherwise, let us assume that one of the $t_{j}$, say $t_{q}$, is greater than $n-1$. We set $B=b_{2} \cdots b_{k} v$ and $c=\left[e_{q}^{\left(t_{q}\right)}, e_{1}^{\left(t_{1}\right)}, \ldots, e_{q-1}^{\left(t_{q-1}\right)}, a_{i}\right]$. It follows from (18) that $c B=\sum_{j} \lambda_{j} e_{q}^{j} c_{j} B$, where each $c_{j}$, as well as $d-c$, can be expressed modulo $\left(L^{m}\right)^{2}$ as a linear combination of commutators $\left[e_{1}^{\left(s_{1}\right)}, \ldots, e_{q}^{\left(s_{q}\right)}, a_{i}\right]$ with $s_{1}+\cdots+s_{q}<t_{1}+\cdots+t_{q}$. By lowering the total degree consecutively we get the inclusion $d B \in V_{k+1}+P_{k}$. The proof is complete.

\section{SUFFICIENT CONDITIONS FOR FINITE GENERATION OF COLOR LIE SUPERALGEBRAS AND THEIR MODULES}

We recall that for any color Lie superalgebra $L$ we have a decomposition of the form $L=L_{+} \oplus L_{-}$.

Lemma 4. Let $L=\sum_{g \in G} L_{g}$ be a finitely generated soluble color Lie superalgebra over a field $F$, char $F \neq 2$, such that for all $g, h \in G$ and $r \in G_{+}$we have an identity of the following form in $L$ :

$$
\left[x, y^{(n)}, z\right]=\sum_{j=1}^{n} \alpha_{j}\left[y^{(j)}, x, y^{(n-j)}, z\right],
$$

where $\alpha_{1}, \ldots, \alpha_{n} \in F, x \in L_{g}, z \in L_{h}$, and $y \in L_{r}$. Then $L_{+}$is a finitely generated color Lie superalgebra and $L_{-}$is a finitely generated. $L_{+}$-module.

Proof. Let $L$ be generated by homogeneous elements $x_{1}, \ldots, x_{m}, y_{1}, \ldots, y_{m}$ with $x_{i} \in L_{-}$and $y_{i} \in L_{+}, i=1, \ldots, m$. We denote by $H$ the subalgebra in $L$ generated by $y_{i}, x_{i}^{2}, i=1, \ldots, m$. Then $H \subset L_{+}$. We denote by $M$ the $H$-submodule in $L$ generated by all commutators of the form $\left[a_{1}, \ldots, a_{r}\right]$, where all the $a_{1}, \ldots, a_{r}$ are commutators of the form

$$
\left[y_{1}^{\left(t_{1}\right)}, \ldots, y_{m}^{\left(t_{m}\right)}, x_{1}^{\left(q_{1}\right)}, \ldots, x_{m}^{\left(q_{m}\right)}, u\right]
$$

such that $u \in\left\{x_{1}, \ldots, x_{m}, y_{1}, \ldots, y_{m}\right\}$ and the following restrictions take place: $0 \leq t_{1}, \ldots, t_{m} \leq n, 0 \leq q_{1}, \ldots, q_{m} \leq 2 n$.

Our next goal is to prove the equation $L=M$. For the elements $a_{\lambda}$ and $a_{\lambda}^{\prime}$ of the form (27) we set $a_{\lambda}<a_{\lambda}^{\prime}$ if and only if $\left(t_{1}, \ldots, t_{m}, q_{1}, \ldots q_{m}\right)$ is lexicographically less than $\left(t_{1}^{\prime}, \ldots, t_{m}^{\prime}, q_{1}^{\prime}, \ldots, q_{m}^{\prime}\right)$ if we compare the components from the right to the left. We extend this partial ordering to all ordered sets of the form $\left\{a_{1}, \ldots, a_{r}\right\}$ by comparing the components from the right to the left.

We want to prove that any commutator $\left[a_{1}, \ldots, a_{r}\right]$ of the elements of the form (27) is in $M$. This will prove $L=M$, because all elements (27) with the restriction $t_{1}+\cdots+t_{m}+q_{1}+\cdots q_{m} \geq 1$ generate $L^{2}$ as a superalgebra. We proceed by induction over the partial ordering.

If the exponents $t_{i}$ and $q_{j}$ of each of the $a_{i}$ do not exceed $n$ and $2 n$ respectively, then $\left[a_{1}, \ldots, a_{r}\right] \in M$ by the construction. Suppose that the inductive hypothesis is satisfied and $a_{i}$ is a commutator of the form (27) with $t_{m}>n$. Then the difference $a_{i}-\left[y_{m}^{\left(t_{m}\right)}, c_{i}\right]$, where $c_{i}=\left[y_{1}^{\left(t_{1}\right)}, \ldots, y_{m-1}^{\left(t_{m-1}\right)}, x_{1}^{\left(q_{1}\right)}, \ldots\right.$, 
$\left.x_{m}^{\left(q_{m}\right)}, u\right]$, can be written in the form of a linear combination of commutators $\left[d_{1}, d_{2}\right]$, where each factor $d_{j}$ has the form (27) and it is strictly less than $a_{i}$. Using identities of the set $(26)$ one can write the commutator $\left[\left[y_{m}^{\left(t_{m}\right)}, c_{i}\right], d\right]$, $d$ being arbitrary homogeneous, as a linear combination of commutators of the form $\left[y_{m}^{(j)},\left[y_{m}^{\left(t_{m}-j\right)}, c_{i}\right], d\right], j \geq 1$. Any $\left[y_{m}^{\left(t_{m}-j\right)}, c_{i}\right]$ is equal to

$$
\left[y_{1}^{\left(t_{1}\right)}, \ldots, y_{m}^{\left(t_{m}-j\right)}, x_{1}^{\left(q_{1}\right)}, \ldots, x_{m}^{\left(q_{m}\right)}, u\right]
$$

plus a linear combination of commutators $\left[d_{1}, d_{2}\right]$, where $d_{1}, d_{2}$ are of the form (27) and $d_{1}, d_{2}<a_{i}$.

Since $y_{m} \in H$, using the Jacobi identity for color Lie superalgebras proves that $\left[a_{1}, \ldots, a_{r}\right]$ lies in the $H$-module generated by commutators of the form $\left[b_{1}, \ldots, b_{s}, a_{i+1}, \ldots, a_{r}\right]$, with $s \geq i$, where all factors have the form (27) with $b_{s}<a_{i}$. Now $\left\{b_{1}, \ldots, b_{s}, a_{i+1}, \ldots, a_{r}\right\}$ is strictly less than $\left\{a_{1}, \ldots, a_{r}\right\}$, hence by the inductive hypothesis, $\left[a_{1}, \ldots, a_{r}\right]$ is in $M$.

Arguing similarly in the case where one of the exponents $t_{1}, \ldots, t_{m}$ in the expression for $a_{i}$ is greater than $n$ or one of the $q_{1}, \ldots, q_{m}$ is greater than $2 n$ and moving $x_{j}^{2}$ to the left, we get $\left[a_{1}, \ldots, a_{r}\right] \in M$ in the general case.

To complete the proof of Lemma 4 it is sufficient to construct a finitely generated subalgebra $B$ of $L_{+}$such that $L_{+}$and $L_{-}$are finitely generated $B$-modules. We use induction over the solubility length of $L$. If $L^{2}=0$ then $B=L_{+}=\left\langle y_{1}, \ldots, y_{m}\right\rangle$. Now suppose that $L^{2} \neq 0$.

Let $R$ denote the subalgebra in $L$ generated by the elements of the form (27) with $0 \leq t_{1}, \ldots, t_{m} \leq n, 0 \leq q_{1}, \ldots, q_{m} \leq 2 n$, and $t_{1}+\cdots+t_{m}+q_{1}+$ $\cdots+q_{m} \geq 1$. Since the solubility length of $R$ is strictly less than that of $L$, it is possible to assume that $R_{+}$contains a finitely generated subalgebra $C$ such that $R_{+}$and $R_{-}$, as $C$-modules, are generated by the set of elements of the form $\left\{Y_{1}, \ldots, Y_{N}\right\},\left\{X_{1}, \ldots, X_{N}\right\}$ respectively.

It follows from $L=M$, proven above, that if

$$
B=\operatorname{alg}\left\{C, y_{1}, \ldots, y_{m}, x_{1}^{2}, \ldots, x_{m}^{2}\right\}
$$

then, as a $B$-module, $L_{+}$is generated by $Y_{i}$ and $y_{i}$, and $L_{-}$by all $X_{i}$ and $x_{i}$, proving Lemma 4 .

In the case of ordinary superalgebras we derive the following result from Lemmas 2, 3, and 4 .

Proposition 2. Let $\mathscr{V}$ be a locally soluble variety of Lie superalgebras over an infinite field $F$, $\operatorname{char} F \neq 2$. Then the following conditions are equivalent.

(a) Any finitely generated Lie superalgebra in $\mathscr{V}$ satisfies the maximality condition for ideals.

(b) Any finitely generated Lie superalgebra $L=L_{0} \oplus L_{1}$ in $\mathscr{V}$ is noetherian as an $L_{0}$-module.

(c) The following identities hold in $\mathscr{V}$ :

$$
\left[x, y^{(n)}, z\right]=\sum_{j=1}^{n} \alpha_{j}^{i}\left[y^{(j)}, x, y^{(n-j)}, z\right],
$$

where $i=1,2,3$. Here if $i=1$ then all three variables $x, y, z$ are in $L_{0}$, if $i=2$ then $x, y \in L_{0}$ and $z \in L_{1}$, and if $i=3$ then $y \in L_{0}$ and $x, z \in L_{1}$. 
Proof. If the third condition holds then $\mathscr{V}$ has an identity

$$
\left[z, y^{(n)}, x\right]=\sum_{j=1}^{n} \beta_{j}\left[y^{(j)}, z, y^{(n-j)}, x\right]
$$

for $z \in L_{1}, x, y \in L_{0}$, and $\beta_{1}, \ldots, \beta_{n} \in F$. Indeed this is another expression of the identity (c) for $i=2$, because $\left[x, y^{(k)}, z\right]$ with an arbitrary $k$ is equal to $(-1)^{k}\left[z, y^{(k)}, x\right]$ modulo the linear span of the commutators $\left[y^{(i)}, z, y^{(n-i)}, x\right], i \geq 1$. It follows from Lemma 4 that both $L_{0}$ and $L_{1}$ are finitely generated $L_{0}$-modules. Applying Lemma 3 we get (b) and this, obviously, implies (a). Now we want to prove that the first condition of the proposition implies the third one. We consider the ideal $I_{k}$ in the $\mathscr{V}$-free algebra $L$ generated by $[x, z],[x, y, z], \ldots,\left[x, y^{(k)}, z\right]$, where $x, y$, and $z$ are free generators of $L$. Then $I_{1} \subset I_{2} \subset \cdots$ and, by the maximality condition, $I_{n}=I_{n-1}$ for some $n$. Hence, $\left[x, y^{(n)}, z\right] \in I_{n-1}$. It follows that

$$
\left[x, y^{(n)}, z\right]=\sum_{j=1}^{n} g_{j}\left[x, y^{(n-j)}, z\right],
$$

where $g_{j}$ is a polynomial depending on ad $x$, ad $y$, and ad $z$. Since $F$ is an infinite field and $x, y, z$ are free generators, it follows that $g_{j}=\beta_{j}(\text { ad } y)^{j}$. Varying the parities of $x, y, z$, without any difficulty we get all three identities as claimed. The proof is complete.

Lemma 5. Let $L=L_{0} \oplus L_{1}$ be a finitely generated soluble Lie superalgebra with identities of the form

$$
\begin{gathered}
{\left[x, y^{(n)}, z\right]=\sum_{j=1}^{n} \alpha_{j}\left[y^{(j)}, x, y^{(n-j)}, z\right], \quad x, y, z \in L_{0},} \\
{\left[x, y^{(n)}, z\right]=\sum_{j=1}^{n} \beta_{j}\left[y^{(j)}, x, y^{(n-j)}, z\right], \quad x, y \in L_{0}, z \in L_{1},} \\
{\left[x, y^{(n)}, z\right]=\sum_{j=1}^{n} \gamma_{j}\left[y^{(j)}, x, y^{(n-j)}, z\right], \quad y \in L_{0}, x, z \in L_{1} .}
\end{gathered}
$$

Suppose also that $M=M_{0} \oplus M_{1}$ is a finitely generated L-module, and the following identities hold for the representation of $L$ on $M$ :

$$
\left[y^{(n)}, z\right] v=\sum_{j=1}^{n} \lambda_{i j} y^{j}\left[y^{(n-j)}, z\right] v,
$$

with $y \in L_{0}, z \in L_{1}, \lambda_{i j} \in F$, and $i=0$ if $v \in M_{0}, i=1$ if $v \in M_{1}$. Then $M$ is a finitely generated module over the superalgebra $H=L_{0} \oplus\left[L_{0}^{c}, L_{1}\right]$ for any $c \geq 2$.

Proof. By Lemma 4 we can say that $L_{0}$ is finitely generated and $L_{1}$, as an $L_{0}$-module, can be generated by a finite set of elements $x_{1}, \ldots, x_{q} \in L_{1}$. Let $\left\{e_{1}, \ldots, e_{m}\right\}$ be a basis of $L_{0}$ modulo $L_{0}^{c}$ chosen in such a way that, modulo $L_{0}^{c},\left[e_{i}, e_{j}\right]$ is equal to a linear combination of elements of the form $e_{k}, k<$ 
$i, j$. Then, modulo $\left[L_{0}^{c}, L_{1}\right], L_{1}$ becomes the linear span of commutators of the form

$$
\left[e_{1}^{\left(t_{1}\right)}, \ldots, e_{m}^{\left(t_{m}\right)}, x_{i}\right]
$$

We order the commutators of the form (32) by comparing vectors $\left(t_{1}, \ldots, t_{m}, i\right)$ lexicographically from the right to the left. Now if $\left\{u_{j}\right\}$ is a finite set of homogeneous generators of $M$ as an $L$-module then, by Poincarè-Birkhoff-Witt's Theorem, $M$ as an $H$-module can be generated by the elements of the form

$$
b_{1} \cdots b_{r} u_{j}, \quad b_{1}>\cdots>b_{r}, r \geq 0,
$$

where $b_{1}, \ldots, b_{r}$ are commutators of the form (32). Let $T$ denote the $H$ submodule of $M$ generated by all elements of the form (32) such that each $b_{j}$ satisfies the conditions $t_{1}, \ldots, t_{m}<n$. To prove our lemma it is sufficient to verify that each element of the form (33) is in $T$. For $r=0$ the above inclusion is obvious. Now suppose that it holds for all numbers less than $r$. Then, by the Jacobi identity, it is sufficient to verify that $T$ contains all elements of the form $b_{1} b_{2} \cdots b_{r} u_{j}$, where the restrictions $t_{1}, \ldots, t_{m}<n$ hold for $b_{2}, \ldots, b_{r}$ and $b_{2}>\cdots>b_{r}$.

Now let $b_{1}=\left[e_{1}^{\left(q_{1}\right)}, \ldots, e_{m}^{\left(q_{m}\right)}, x_{i}\right]$. We consider first the case where $q_{1}, \ldots$, $q_{m}<n$. If $b_{1}>b_{2}$ then $b_{1} \cdots b_{r} u_{j}$ is in $T$ by construction. If $b_{i}>b_{1} \geq b_{i+1}$, then applying induction over $r$ shows that $b_{1} \cdots b_{r} u_{j}$ is congruent, modulo $T$, to $B=b_{2} \cdots b_{i} b_{1} b_{i+1} \cdots b_{r} u_{j}$. In the case where $b_{1} \neq b_{i+1}$ we find that $B$ is in $T$, otherwise, if $b_{1}=b_{i+1}$, we have $B \in T$ by the induction hypothesis, since $b_{1}^{2} \in L_{0} \subset H$.

Now let the exponents $q_{1}, \ldots, q_{m}$, in the expression of $b_{1}$, be arbitrary. We set $b_{2} \cdots b_{r} u_{j}=v$. If $q_{1} \geq n$ then, using identity (31), we can write $b_{1} v$ as a linear combination of elements of the form $e_{1}^{j} c_{j} v$, where $c_{j}$ is a commutator of the form (32) with $t_{1} \leq n-1$. If $q_{j} \geq n$ and $d=\left[e_{j}^{\left(q_{j}\right)}, e_{1}^{\left(q_{1}\right)}, \ldots, e_{m}^{\left(q_{m}\right)}, x_{i}\right]$ then the difference $b_{1}-d$ can be expressed, modulo $H$, as a linear combination of elements of the form (32) which are strictly less than $b_{1}$. This enables us to lower the degree of $b_{1}$ with respect to any of the variables $e_{j}$ using (31). So, all reduces to the situation considered above, and the proof is complete.

\section{Finite dimeNSION OF CERTAIN MOdULES OVER LIE SUPERALGEBRAS}

All results that follow from now on refer to Lie superalgebras over a field $F$ of characteristic different from 2.

Lemma 6. Let $L, M$, and $H$ be as in Lemma 5. If $Q$ is an infinite-dimensional $H$-submodule in $M$ and $\operatorname{dim} M / Q<\infty$, then $Q$ contains a nonzero $L$ submodule.

Proof. Let $\left\{e_{1}, \ldots, e_{m}\right\}$ be a basis of $L_{0}$ modulo $L_{0}^{c}$ and $\left\{x_{i}\right\}$ a finite set of elements, generating $L_{1}$ as an $L_{0}$-module. Let $\left\{z_{1}, \ldots, z_{N}\right\}$ be the set of all commutators of the form

$$
\left[e_{1}^{\left(q_{1}\right)}, \ldots, e_{m}^{\left(q_{m}\right)}, x_{i}\right], \quad 0 \leq q_{1}, \ldots, q_{m} \leq n-1 .
$$

We denote by $\left\{A_{1}, \ldots, A_{T}\right\}$ the set of all linear operators on $M$ of the form $z_{i_{1}} \cdots z_{i_{k}}, N \geq i_{1}>\cdots>i_{k} \geq 1$. By our hypothesis we can choose in $Q$ an infinite-dimensional subspace $W_{1}$ such that $A_{1} W_{1} \subset Q$. Now we can choose in 
$W_{1}$ an infinite-dimensional subspace $W_{2}$ such that $A_{2} W_{2} \subset Q$. By repeating this procedure we will arrive at a nonzero vector $v$ in $Q$ such that $A_{i} v \in Q$ for all $i=1, \ldots, T$.

Now let $U(L)$ be the enveloping algebra for $L$ and let $P$ be a subspace in $U(L)$ spanned by the elements of the form $f A_{i}, i=0, \ldots, T$, with $A_{0}=1$ and $f \in U(H)$. Then $P v \subset Q$ by the choice of $v$. The proof of our lemma will be complete if we verify that $P v$ is an $L$-module. Since $H=L_{0} \oplus\left[L_{0}^{c}, L_{1}\right]$, it is sufficient to verify the inclusion $a P v \subset P v$, where $a$ is a commutator of the form (32). As in the proof of Lemma 5 one can show that identity (31) implies the following relation:

$$
a w \equiv \sum_{j} h_{j} z_{j} w \quad(\bmod U(H) w),
$$

where $a$ and $w$ are arbitrary in $L$ and $M$ respectively, each $z_{j}$ is a commutator of the form (34), and $h_{j} \in U(H)$. It follows from (35) that, to finish the proof, it remains to show that $P v$ contains all elements of the form $z_{j} A_{i} v$. Let $A_{i}=z_{j_{1}} \cdots z_{j_{k}}$. If $k=1$ then $\left[z_{j}, A_{i}\right] \in L_{0}$, hence it is obvious that $z_{j} A_{i} v \in P v$. Now suppose $k>1$. If $j>j_{1}$ then the product $z_{j} A_{i}$ is one of the operators $A_{1}, \ldots, A_{T}$, hence $z_{j} A_{i} v \in P v$. Otherwise, if $j_{s-1}>j \geq j_{s}$ for some $s$ then moving $z_{j}$ to the right in the expression of $z_{j} A_{i} v$ we can write this latter, modulo $P v$, as the sum of some of the products of the form

$$
z_{j_{1}} \cdots z_{j_{r}} g_{r} z_{j_{r+2}} \cdots z_{j_{k}} v
$$

in which $g_{r} \in L_{0}, r \leq s-1$. Moving $g_{r}$ to the left we get an expression for any of (36) as the sum of some elements of the form $b_{t}=f_{t} z_{i_{t}} \cdots z_{i_{k}}$ with $i_{t}>i_{t+1}>\cdots>i_{k}, 2 \leq t \leq k, f_{t} \in L$. Hence, considering (35), we express $z_{j} A_{i} v$ modulo $P v$ as the sum of elements of the form $h z_{l} A_{l} v$ with $h \in U(H)$, $A_{l}=z_{i_{1}} \cdots z_{i_{s}}$, and $s \leq k-1$. Applying induction over $k$ we derive that all these elements are in $P v$, proving the lemma.

Lemma 7. Let $L$ and $M$ be as in Lemma 5 and the action of $L_{0}^{c}$ on $M$ and $L_{1}$ is nilpotent. Also let $c=2$ if the characteristic of the ground field is zero. If $M$ is an irreducible L-module then $\operatorname{dim} M<\infty$.

Proof. We denote $L_{1}$ by $S_{0}$ and $\left[L_{0}^{c}, S_{i-1}\right]$ by $S_{i}$, if $i>0$. Then $S_{d}=0$ for some $d$. We proceed by induction over $d$. If $d=1$ then, using the notation in Lemmas 5 and $6, H=L_{0}$. If $M$ is an irreducible $H$-module then $H^{c} M=0$, hence $M$ is an irreducible module over the finite-dimensional Lie algebra $H / H^{c}$, and $c=2$ if $\operatorname{char} F=0$. By Proposition $1, \operatorname{dim} M<\infty$.

If $M$ has a nonzero proper $H$-submodule then it has a maximal such submodule. We denote this latter by $Q$. Then $\operatorname{dim} M / Q<\infty$ and using Lemma 6 guarantees the finiteness of the dimension of $M$.

Now suppose $d>1$. Then $H=L_{0} \oplus\left[L_{0}^{c}, L_{1}\right]$ is a finitely generated Lie superalgebra by Proposition 2. By Lemma 5, M is a finitely generated $\mathrm{H}$ module. Hence, by the induction hypothesis, all irreducible submodules and quotient-modules of $M$ as a $H$-module are finite dimensional.

Let $Q$ be a maximal $H$-submodule of $M, Q \neq M$. Then $\operatorname{dim} M / Q<\infty$ and Lemma 6 complete the proof of Lemma 7.

Lemma 8. Let $L=L_{0} \oplus L_{1}$ and $M=M_{0} \oplus M_{1}$ be as in Lemma 7 and the action of $L_{0}$ on $M_{0}$ as well on $M_{1}$ satisfies the identities of the form (18). Also let 
$M$ have a finite-dimensional $L$-submodule $K$ which has a nonzero intersection with any nonzero L-submodule. Then $\operatorname{dim} M<\infty$.

Proof. First we consider the case where $L_{1}=0$. If $L_{0}^{c} M=0$ then $M$ is a finitely generated module over a finite-dimensional Lie algebra $L / \operatorname{Ann}_{L}(M)$. It follows by Lemma 1 that the semidirect product $\left(L / \operatorname{Ann}_{L}(M)\right) \oplus M$ (where $L / \operatorname{Ann}_{L}(M)$ is a subalgebra and $M$ is an ideal) is finite dimensional, hence we will assume in what follows that $L_{0}^{c} M=T \neq 0$. Now Lemma 3 applies to both $\left(L_{0}, M_{0}\right)$ and $\left(L_{0}, M_{1}\right)$, hence $T$ is a finitely generated $L$-module. In this case $L, T$, and $T \cap K$ satisfy the hypotheses of the lemma, while the nilpotent index of the action of $L_{0}^{c}$ on $T$ is by one less than in the case of $(L, M)$. Therefore, the induction argument enables us to conclude that $T$ is finite dimensional.

Now let $D_{j}$ denote the $L$-submodule in $M$ consisting of those $v$ for which one has $b_{1} \cdots b_{j} v=0$ for any $b_{1}, \ldots, b_{j} \in L^{c}$. We want to verify that all $D_{j}$ have finite codimension in $M$. By our hypothesis, $D_{q}=M$ for some $q$. Suppose the finiteness of the dimension of $M / D_{j+1}$ has been proven. Let $A_{1}, \ldots, A_{N}$ be all the operators of End $M$ of the form $z_{1} \cdots z_{j}$, where each factor is of the form

$$
\left[e_{1}^{\left(t_{1}\right)}, \ldots, e_{m}^{\left(t_{m}\right)}, y_{i}\right]
$$

with $0 \leq t_{1}, \ldots, t_{m} \leq n-1,\left\{e_{1}, \ldots, e_{m}\right\}$ being a basis of $L$ modulo $L^{c}$, and $\left\{y_{i}\right\}$ a finite set of elements generating $L^{c}$ as an ideal of $L$.

Set $B_{j}=\operatorname{ker} A_{j}$. Since $A_{j} M \subset T$ and $\operatorname{dim} T<\infty$, the codimension of $B_{j}$ in $M$ is finite. Then also for $Q=B_{1} \cap \cdots \cap B_{N} \cap D_{j+1}$ we have $\operatorname{dim} M / Q<\infty$. Now we want to show that $D_{j} \supset Q$. If $w \in Q$ then it is sufficient to verify $a_{1} \cdots a_{j} w=0$ with $a_{1}, \ldots, a_{j}$ of the form (37) without any restrictions on the exponents $t_{1}, \ldots, t_{m}$.

For instance, let $a_{r}=\left[e_{1}^{\left(q_{1}\right)}, \ldots, e_{m}^{\left(q_{m}\right)}, y_{i}\right]$ and $q_{s} \geq n$. Since $w \in D_{j+1}$ the factor $a_{r}$ in $a_{1} \cdots a_{j} w$ can be replaced by $b_{r}$ and by a linear combination of $a_{r}^{\prime}$ of the same form but also with $q_{1}^{\prime}+\cdots+q_{m}^{\prime}<q_{1}+\cdots+q_{m}$. Here $b_{r}$ is the commutator of the form $\left[e_{s}^{\left(q_{s}\right)}, e_{1}^{\left(q_{1}\right)}, \ldots, e_{s-1}^{\left(q_{s-1}\right)}, e_{s+1}^{\left(q_{s+1}\right)}, \ldots, e_{m}^{\left(q_{m}\right)}, y_{i}\right]$. Using (18) we can replace this latter by a linear combination of the products of the form $e_{s}^{k} c_{r}$ in which any $c_{r}$ is a commutator of the form (37) with $t_{1}+\cdots+t_{m}<q_{1}+\cdots+q_{m}$. This means that $a_{1} \cdots a_{j} w$ belongs to the $L$-submodule generated by $b_{1} \cdots b_{j} w$ with $b_{1}, \ldots, b_{j}$ of the form (37) with bounded exponents $e_{1}, \ldots, e_{m}$. Hence $a_{1} \cdots a_{j} w=0$ and the proof of $Q \subset D_{j}$ is complete.

Now from the finiteness of the dimension of $M / D_{j}$ it follows that, for $j=1$, $M$ has a submodule $D_{1}$ of finite codimension such that $L^{c} D_{1}=0$. If $D_{1} \neq 0$ then $L, D_{1}$, and $D_{1} \cap K$ satisfy the hypothesis of the lemma, hence, as shown above, $\operatorname{dim} D_{1}<\infty$. Thus, in the case $L=L_{0}, M$ is a finite-dimensional $L$-module.

Now suppose $L_{1} \neq 0$. We make use of the nilpotence of the action of $L_{0}^{c}$ on $L_{1}$ and proceed by induction over the nilpotent index. We set $H=$ $L_{0} \oplus\left[L_{0}^{c}, L_{1}\right]$. By Lemma $5, M$ is finitely generated as an $H$-module and it follows by Proposition 2 that $H$ is a finitely generated Lie superalgebra. By Zorn's Lemma there is a maximal $H$-submodule $Q$ in $M$ whose intersection with $K$ is trivial. Then $H, M / Q$, and $K+Q / Q$ satisfy the hypotheses of 
the lemma. The induction hypothesis (the induction starts with the above case $L_{1}=0$ ) enables us to assume that $M / Q$ is finite dimensional. If $Q$ is infinite dimensional then, by Lemma 6 , there exist a nonzero $L$-submodule $W$ in $Q$. Then $W \cap K=0$ by the choice of $Q$ which contradicts the hypotheses of the lemma. Consequently, $\operatorname{dim} Q<\infty$ and the proof of Lemma 8 is complete.

\section{LOCALLY RESIDUALLY FINITE VARIETIES OF LIE SUPERALGEBRAS}

Theorem 4. Le $\mathscr{V}$ be a locally soluble variety of Lie superalgebras over an infinite field $F$ of characteristic different from 2. Then the property of being locally residually finite for $\mathscr{V}$ is equivalent to the following two conditions:

(a) $\mathscr{V}$ satisfies the identities of the form (28), (29), (30).

(b) If $\operatorname{char} F=0$, then any finitely generated algebra $L=L_{0} \oplus L_{1}$ in $\mathscr{V}$ satisfies an identity of the form

$$
\left[\left[x_{1}, y_{1}\right], \ldots,\left[x_{m}, y_{m}\right], z\right]=0, \quad x_{i}, y_{i} \in L_{0}, z \in L .
$$

Proof. We prove first that the conditions above are necessary. We have obtained (a) in Theorem 3 for arbitrary locally residually finite varieties.

Now suppose char $F=0$ and let $L=L_{0} \oplus L_{1}$ be a free algebra in $\mathscr{V}$ with free generators of the form $x_{1}, \ldots, x_{t}, y_{1}, \ldots, y_{t}, z_{1}, z_{2}$, where $z_{2} \in L_{1}$ and $z_{1}$ and all the $x_{i}, y_{i}$ are in $L_{0}$. Following the argument in Lemma 5 in [5] virtually verbatim we may assume that $f^{N}=0$ for some $N$, where $f=$ $\operatorname{ad}\left(\left[y_{1}, x_{1}\right]+\cdots+\left[y_{t}, x_{t}\right]\right)$. If $b$ is an arbitrary element in $L_{0}^{2}$, depending only on the variables in $y_{1}, \ldots, y_{t}$, then $b$ can be written in the form $b=\left[y_{1}, b_{1}\right]+$ $\cdots+\left[y_{t}, b_{t}\right]$, where $b_{1}, \ldots, b_{t} \in L_{0}$. Then the mapping $x_{i} \mapsto b_{i}$ takes $f^{N} z_{j}$, which is zero, into the commutator $\left[b^{(N)}, z_{j}\right], j=1,2$. Hence, if we denote by $H$ the intersection of $L_{0}^{2}$ with the subalgebra generated by $y_{1}, \ldots, y_{t}$ we will have a weak identity $x^{N}=0$ satisfied in the representation of $H$ by linear operators of $L_{0}$ and $L_{1}$. (Let $A$ be an associative enveloping algebra of the Lie algebra $B$. This means that $B \subset A$ and elements of $B$ generate $A$ as an associative algebra. If the associative polynomial $f\left(x_{1}, \ldots, x_{n}\right)$ is equal to zero in $A$ for any $x_{1}, \ldots, x_{n} \in B$ then this polynomial is called a weak identity of the pair $(A, B)$.)

We shall prove that if $H$ is a Lie algebra of linear transformations on the vector space $W$ over a field $F$ of characteristic zero and $x^{N}=0$ for any $x \in H$, then $x_{1} \cdots x_{k}=0$ as a linear map on $W$ for some $k$, where $x_{1}, \ldots, x_{k}$ are arbitrary elements of $H$.

By Theorem 4.1 in [12] $H$ is a nilpotent Lie algebra, since $(\operatorname{ad} x)^{2 N+1}=0$. Let us prove our statement for an abelian algebra $H$.

Since $[H, H]=0$, the unique monomial which depends on all $b_{1}, \ldots, b_{N}$ on the left-hand side of equation $\left(b_{1}+\cdots+b_{N}\right)^{N}=0$ is equal to $N ! b_{1} \cdots b_{N}$. Since char $F=0$, it follows that $b_{1} \cdots b_{N}=0$, and the proof of the statement is complete for an abelian algebra.

Now suppose $H^{t}=0, t \geq 2$. We proceed by induction over $t$. Denote by $Z$ the center of $H$. Then $Z$ acts on $W$ as a nilpotent space of transformations. We may construct a finite chain of $H$-submodules

$$
W=W_{0} \supset W_{1} \supset \cdots \supset W_{j} \supset \cdots \supset W_{N}=0,
$$

where $W_{j}=Z W_{j-1}$ for $j=1, \ldots, N$. For any $j \geq 0, M_{j}=W_{j} / W_{j+1}$ is an $H / Z$-module and, by the inductive hypothesis, $H$ acts on $M_{j}$ in a nilpotent 
way, since $(H / Z)^{t-1}=0$. Hence, $H$ is a nilpotent space of transformations on $W$, and the proof of the statement is complete.

This implies the nilpotence of the action of $H$ on $L$, hence the validity of (38) since, by Theorem 3 and Lemma $4, L_{0}$ is finitely generated.

Now we want to prove that the conditions of our theorem are sufficient. Let $L$ be a finitely generated soluble Lie superalgebra over a field $F$ with (28), (29), (30) and, if char $F=0$, with (38). It follows from (28) and (29) that the representations of $L_{0}$ in $L_{0}$ and $L_{1}$ satisfy the identities of the form (18) and (19). Similarly, it follows from (29) and (30) that the adjoint representation of $L$ satisfies (31). It was shown in the proof of Proposition 2 that $L$ satisfies all the conditions of Lemma 4 . Hence, $L_{0}$ is a finitely generated algebra and, by Lemma 2, there exist such $c$ that $L_{0}^{c}$ acts on $L$ as a nilpotent space of operators. One may assume that $c=2$ is $\operatorname{char} F=0$. As in the proof of Theorem 2 it is sufficient to show that if $L$ has the least nonzero ideal $K$, then it is finite dimensional. Since $L$ as a module over itself and $K$ as an $L$-module satisfy the hypotheses of Lemmas 5,7 , and 8 , it follows by Lemma 7 that $\operatorname{dim} K<\infty$ and by Lemma 8 that $\operatorname{dim} L<\infty$. Now the proof is complete.

It is possible to abandon the solubility condition in Theorem 4 in the case where the ground field is of characteristic zero.

Lemma 9. Let $L=L_{0} \oplus L_{1}$ be a Lie superalgebra over a field of characteristic zero satisfying (28), (29), and (30), and, for each $p$, let there exist an $N$ such that $\left(\operatorname{ad}\left(\left[a_{1}, b_{1}\right]+\cdots+\left[a_{p}, b_{p}\right]\right)\right)^{N}=0$ for all $b_{1}, \ldots, b_{p}, a_{1}, \ldots, a_{p} \in L_{0}$. Then $L$ is locally soluble.

Proof. We construct a subalgebra in $L$ of the form

$$
R=R_{0} \oplus R_{1}=L_{0} \oplus\left[L_{0}, \ldots, L_{0}, L_{1}\right]
$$

such that for some $k$ we have $\left(\operatorname{ad} b^{2}\right)^{k+1}=0$ in $R$ for any $b$ in $R_{1}$. First consider the case where not all the $\beta_{j}$ in (29) and the $\gamma_{j}$ in (30) are zero.

We can divide variables in (29) and (30) using (29) in the same way as was done in Lemma 2. This gives the identities of the form

$$
\begin{gathered}
{\left[x, y^{(k)}, Z\right]=\sum_{j=1}^{k} \lambda_{j}\left[y^{(j)}, x, y^{(k-j)}, Z\right], \quad x, y \in L_{0},} \\
{\left[x, y^{(k)}, Z\right]=\sum_{j=1}^{k} \mu_{j}\left[y^{(j)}, x, y^{(k-j)}, Z\right], \quad y \in L_{0}, \quad x \in L_{1} .}
\end{gathered}
$$

Here, $Z=\left[t^{(r)}, z\right], z \in L_{1}, t \in L_{0}$ and some $r$. Besides, $\lambda_{k} \neq 0$ and $\mu_{k} \neq 0$.

Let $M$ denote the $L_{0}$-submodule in $L_{1}$ generated by all elements of the form $\left[a^{(r)}, b\right], a \in L_{0}, b \in L_{1}$. Then the representation of $L_{0}$ in $L_{1} / M$ satisfies the weak identity $u^{r}=0$, whence we derive the nilpotence of the action of $L_{0}$ on $L_{1} / M$ (it was shown in the proof of Theorem 4). Consequently, for some $s$, we have

$$
T_{s} \subset M,
$$

where $T_{0}=L_{1}$ and $T_{j}=\left[L_{0}, T_{j-1}\right]$ if $j>0$. We want to show that $M$ is the linear span of the elements of the form $\left[a^{(r)}, b\right], a \in L_{0}, b \in L_{1}$. If $a, d \in L_{0}$ 
and $b \in L_{1}$, then

$$
\left[d, a^{(r)}, b\right]-\left[a^{(r)}, d, b\right]=\sum\left[a^{(j)},[d, a], a^{(r-j-1)}, b\right] .
$$

The sum on the right-hand side of $(42)$ is one of the values of a partial linearization of $\left[y^{(r)}, x\right], y \in L_{0}, x \in L_{1}$. Hence, $\left[d, a^{(r)}, b\right]$ can be written as a linear combination of values of the monomial $\left[y^{(r)}, x\right]$ in $L$. It follows then that $M$ is the linear span of elements of the form $\left[a^{(r)}, b\right], a \in L_{0}, b \in L_{1}$.

Now let $R=R_{0} \oplus R_{1}$ be the subalgebra $L_{0} \oplus T_{s}$. Suppose $a \in R_{0}, b \in R_{1}$, and $[a, b]=0$. Since $R_{1} \subset M$, it follows from (39) and (40) with $x=c$, $y=a$, and $z=b$ that $\left[a^{(k)}, c, b\right]=0$ for any $c \in R$. Replacing $a$ by $b^{2}$ in this relation we get $(\operatorname{ad} b)^{2 k+1} z=0$. It follows from this that, for any $b$ in $R_{1}$, we have $\left(\operatorname{ad} b^{2}\right)^{k+1}=0$.

If all the $\beta_{j}$ in (29) are zero the the weak identity $x^{n+1}=0$ holds in the representation of $L_{0}$ on $L_{1}$, hence $T_{s}=0$ for some $s$ and $R_{1}=0$. If the coefficients $\beta_{j}$ are not equal to zero simultaneously then we have (39). As was shown, $\left(\operatorname{ad~} b^{2}\right)^{k+1}$ is the zero map on $R_{0}$ for any $b$ in $R_{1}$. If, in addition, all the $\gamma_{j}$ in (30) are zero then we have $\left[x,\left(x^{2}\right)^{(n)}, z\right]=0$ for any $x, z$ in $L_{1}$. Hence, $\left(\operatorname{ad} b^{2}\right)^{(n+1)}=0$ on $L_{1}$ for arbitrary $b$ in $L_{1}$.

Let $a_{1}, \ldots, a_{q}$ be arbitrary elements in $R_{0}$ such that $\left(\operatorname{ad} a_{j}\right)^{T}=0$ in $R$. We want to show that there exists such a $P=P(q, T)$ that $\left(\operatorname{ad}\left(a_{1}+\cdots+a_{q}\right)\right)^{P}$ $=0$ in $R$. If $H$ is the Lie algebra generated by $a_{1}, \ldots, a_{q}$ then the hypothesis of the lemma there exists a $N$ such that $\left[b^{(N)}, u\right]=0$ for all $u \in R$ and $b \in H^{2}$. It follows from this that $H^{2}$ acts on $R$ in a nilpotent way, that is, there exists $m$, depending only on $N$, such that $\left[d_{1}, \ldots, d_{m}, u\right]=0$ for $d_{1}, \ldots, d_{m} \in H^{2}$ and $u \in R$. As a Lie algebra, $H^{2}$ is generated by the commutators of the form

$$
\left[a_{1}^{\left(t_{1}\right)}, \ldots, a_{q}^{\left(t_{q}\right)}, a_{i}\right], \quad 0 \leq t_{1}, \ldots, t_{q} \leq T-1, \sum t_{i}>0 .
$$

Now $\left(\operatorname{ad}\left(a_{1}+\cdots+a_{q}\right)\right)^{P}(v)$ can be written as a linear combination of commutators of the form

$$
\left[b_{1}, \ldots, b_{r}, a_{1}^{\left(j_{1}\right)}, \ldots, a_{q}^{\left(j_{q}\right)}, v\right], \quad 0 \leq j_{1}, \ldots, j_{q} \leq T-1,
$$

where $b_{1}, \ldots, b_{r}$ have the form (43). Since the degree of each element of the form (43) over all variables $a_{i}$ does not exceed $q T$, we have $r \geq P / q T-1$. It follows then that $\left(\operatorname{ad}\left(a_{1}+\cdots+a_{q}\right)\right)^{P}=0$ on $R$ if $P \geq(m+1) q T$.

It follows from what has been proved that there exists a $t$ such that for any $b_{1}, b_{2} \in R_{1}$ we have $\left(\operatorname{ad}\left[b_{1}, b_{2}\right]\right)^{t}=0$ since $2\left[b_{1}, b_{2}\right]=\left(b_{1}+b_{2}\right)^{2}-b_{1}^{2}-b_{2}^{2}$. In its turn, this means that the action of any element in $R_{1}^{2}$ on $R$ is nilpotent.

Now we can prove the local solubility of the Lie superalgebra $H=H_{0} \oplus H_{1}=$ $R_{1}^{2} \oplus\left[R_{0}, R_{1}\right]$. Let $S=S_{0} \oplus S_{1}=\operatorname{alg}\left\{y_{1}, \ldots, y_{q}, x_{1}, \ldots, x_{q}\right\}$ be a finitely generated subalgebra in $H$, where $y_{i} \in H_{0}$ and $x_{i} \in H_{1}$. We will first prove the nilpotence of the action of $S_{0}$ on $S_{1}$.

If $b \in S_{0}$, then $b=a_{1}+\cdots+a_{q}+b_{1}+\cdots+b_{q}+y$, where $b_{i}=\left[z_{i}, x_{i}\right]$, $z_{i} \in H_{1}, a_{i}=\left[t_{i}, y_{i}\right], t_{i} \in H_{0}$, and $y$ is a linear combination of generators $y_{1}, \ldots, y_{q}$. It follows from the nilpotence of the action of $R_{1}^{2}$ on $R$ that there exists such an $N_{0}$ that $\left(\operatorname{ad} y_{i}\right)^{N_{0}}=0, i=1, \ldots, q$. Since $\left(\operatorname{ad} b_{i}\right)^{t}$ $=0$ there exists an $N_{1}$, which does not depend on $b_{1}, \ldots, b_{q}$, such that $\left(\operatorname{ad}\left(b_{1}+\cdots+b_{q}\right)\right)^{N_{1}}=0$. By the hypothesis of the lemma there exists an $N_{2}$, 
depending only on $q$, such that $\left(\operatorname{ad}\left(a_{1}+\cdots+a_{q}\right)\right)^{N_{2}}=0$. It follows that $(\operatorname{ad} b)^{N}=0$ in $S$, and that $N$ does not depend on $b$. Consequently $S_{0}$ acts on $S_{1}$ in a nilpotent way. Since $S^{2} \subset S_{0} \oplus\left[S_{0}, S_{1}\right]$, it follows from this that for some $j$ we have $S^{(j)} \subset S_{0}$, where $S^{(j)}$ is the $j$ th term of the derived series of $S$. By our hypothesis, $S_{0}$ satisfies (28), which implies solubility (see [6]). Hence $S$ is a soluble Lie superalgebra.

It follows from the definition of $R$ and from (41) that $L^{(s)} \subset R$. Now $R$ has an ideal $H$ such that the derived algebra of $R / H$ is an ordinary Lie algebra since $R^{2} \subset H+R_{0}$. As $(R / H)^{2}$ is a Lie algebra with (28), it is soluble. Hence, $R^{(N)} \subset H$ for some $N$. It follows then that $L^{(q)} \subset H$ for some $q$.

So far we have proved the local solubility of $L^{(q)}$, an ideal of the Lie superalgebra $L$. To derive the local solubility of $L$ we need two auxiliary lemmas.

Lemma 10. Let $L=L_{0} \oplus L_{1}$ be a finitely generated Lie superalgebra satisfying the conditions of Lemma 9 with locally soluble derived algebra. Then $L_{0}$ is a finitely generated Lie algebra.

Proof. If $L$ is a Lie superalgebra generated by even elements $y_{1}, \ldots, y_{m}$ and odd elements $x_{1}, \ldots, x_{m}$ then this is the linear span (modulo alg $\left\{y_{1}, \ldots, y_{m}\right\}$ ) of the commutators of the form $\left[b_{1}, \ldots, b_{r}\right]$ such that each factor is a commutator of the form $\left[y_{j_{1}}, \ldots, y_{j_{s}}, x_{i}\right]$. Each commutator of this kind can be replaced by a linear combination of elements of the form

$$
\left[y_{1}^{\left(\lambda_{1}\right)}, \ldots, y_{m}^{\left(\lambda_{m}\right)}, h_{1}, \ldots, h_{t}, x_{i}\right]
$$

where $h_{1}, \ldots, h_{t}$ are commutators of the form

$$
\left[y_{1}^{\left(\mu_{1}\right)}, \ldots, y_{m}^{\left(\mu_{m}\right)}, y_{j}\right], \quad \mu_{1}+\cdots+\mu_{m}>0 .
$$

If $H_{0}=\operatorname{alg}\left\{y_{1}, \ldots, y_{m}\right\}$ then, by the hypothesis, $H_{0}^{2}$ acts on $L$ in a nilpotent way, hence $t$ in (44) does not exceed a certain number $t_{0}$.

Using (28) and (29), as was done in Lemma 5, it is easy to observe that $\left[b_{1}, \ldots, b_{r}\right]$ is in the $H_{0}$-submodule generated by the commutators of the form $\left[c_{1}, \ldots, c_{r}\right]$, where each $c_{i}$ has the form (44), (45) with $\lambda_{1}, \ldots, \lambda_{m}$, $\mu_{1}, \ldots, \mu_{m} \leq n-1$. The number of these elements is finite. If we denote these elements by $z_{1}, \ldots, z_{p}$ then $L$ coincides with the $H_{0}$-submodule generated by $B+H_{0}$, where $B=B_{0} \oplus B_{1}=\operatorname{alg}\left\{z_{1}, \ldots, z_{p}\right\}$. Since all the $z_{i}$ are in $B_{1}$, the derived algebra $B^{2}$ of $B$ is generated by a finite number of commutators of the form $\left[z_{1}^{\left(\gamma_{1}\right)}, \ldots, z_{p}^{\left(\gamma_{p}\right)}, z_{i}\right]$ with $0 \leq \gamma_{1}, \ldots, \gamma_{p} \leq 2, \sum \gamma_{i}>0$.

Since $L^{2}$ is locally soluble and $B^{2} \subset L^{2}, B$ is a soluble Lie superalgebra satisfying the hypotheses of Lemma 4 . Hence, $B_{0}$ is a finitely generated Lie algebra. Since $L_{0}=\operatorname{alg}\left\{y_{1}, \ldots, y_{m}, B_{0}\right\}$, it follows that the proof of Lemma 10 is complete.

Lemma 11. Let $L$ be a Lie superalgebra satisfying the conditions of Lemma 9. If $L^{2}$ is a locally soluble Lie superalgebra then $L$ is locally soluble.

Proof. Let $L$ be finitely generated. Then, by Lemma 10, its even component $L_{0}$ is finitely generated, too. By the hypothesis of Lemma $9, L_{0}^{2}$ acts on $L$ in a nilpotent way.

As before, $L^{(q)} \subset H$, where $H=R_{1}^{2} \oplus\left[R_{0}, R_{1}\right]$. If $d=\operatorname{dim} L_{0} / L_{0}^{2}$ then any element $h$ in $H_{0}$ can be written as the sum of at most $d+1$ summands, one of them being in $L_{0}^{2}$, while the remaining have the form $\left[a_{1}, a_{2}\right]$, where 
$a_{1}, a_{2} \in R_{1}$. Since $\left(\operatorname{ad}\left[a_{1}, a_{2}\right]\right)^{t}=0$ there exists a $p$ such that $(\operatorname{ad} h)^{p}=0$, $p$ not depending on $h \in H_{0}$. It follows then that the action of $H_{0}$ on $H_{1}$ is nilpotent and that $H$ is soluble (as in the proof of Lemma 9). Since $L^{(q)} \subset H$, $L$ is soluble, proving the lemma.

To finish the proof of Lemma 9 it is sufficient to recall that $L^{(q)}$ is locally soluble for some $q$. Now Lemma 11 enables us to derive the solubility of $L^{(q-1)}, L^{(q-2)}, \ldots, L$, and the proof is complete.

Theorem 5. A variety $\mathscr{V}$ of Lie superalgebras over a field of characteristic zero is locally residually finite if and only if it satisfies identities of the form (28), (29), and (30), and, in any finitely generated algebra of $\mathscr{V}$, we have an identity of the form (38).

Proof. If a variety $\mathscr{V}$ satisfies all identities listed above then, by Lemma 9 , it is locally soluble. By Theorem $4, \mathscr{V}$ is a locally residually finite variety.

Now let $\mathscr{V}$ be a locally residually finite variety of Lie superalgebras. By Theorem 3 then (28), (29), and (30) hold. As in Theorem 4, for even free generators $x_{1}, \ldots, x_{t}, y_{1}, \ldots, y_{t}$ of a relatively free algebra in $\mathscr{V}$, there exists a number $N$ such that $\left(\operatorname{ad}\left(\left[x_{1}, y_{1}\right]+\cdots+\left[x_{t}, y_{t}\right]\right)\right)^{N}=0$. Consequently, any finitely generated algebra in $\mathscr{V}$ is soluble by Lemma 9 and, as was shown in Theorem 4, it satisfies an identity of the form (38). Now the proof of Theorem 5 is complete.

\section{REFERENCES}

1. Yu. A. Bahturin and V. S. Drensky, Identities of soluble colour Lie superalgebras, Algebra i Logika 26 (1987), 403-418. (Russian)

2. A. A. Mikhalev, Subalgebras of colour Lie superalgebras, Mat. Zametki 37 (1985), 653-662. (Russian)

3. M. A. Vasiliev, De Sitter supergravity with positive constant and generalized Lie superalgebras, Classical Quantum Gravity 2 (1985), 645-662.

4. Yu. A. Bahturin, On residual properties of Lie algebras, Mat. Zametki 12 (1972), 713-716. (Russian)

5. M. V. Zaicev, Locally residually finite varieties of Lie algebras, Mat. Zametki 44 (1988), 352-361. (Russian)

6. __ Residual finiteness and Noetherian property of finitely generated Lie algebras, Mat. Sbornik 136 (1988), 500-509. (Russian)

7. M. Scheunert, The theory of Lie superalgebras. An introduction. Lecture Notes in Math., vol. 716, Springer-Verlag, 1979.

8. C. W. Curtis, Noncommutative extensions of Hilbert rings, Proc. Amer. Math. Soc. 4 (1953), 945-955.

9. I. B. Volichenko, On the varieties of the center-by-metabelian Lie algebras, Inst. Math. Akad. Nauk Byelorussian SSR, Preprint No. 16(96), 1980.

10. S. P. Mishchenko, Structure and identities of certain soluble Lie algebras, Ph.D. Thesis, Moscow University, 1982. (Russian)

11. Yu. A. Bahturin, Identical relations in Lie algebras, VNU Science Press, Utrecht, 1987.

12. A. I. Kostrikin, Around Burnside, Springer-Verlag, 1990.

Department of Algebra, Faculty of Mathematics and Mechanics, Moscow State UniVERSITY, MOSCOW 119899, RUSSIA 